\title{
Mouse subthalamic nucleus neurons with local axon collaterals
}

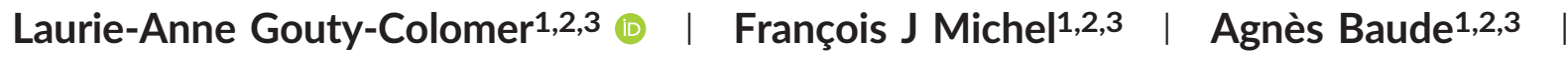 \\ Catherine Lopez-Pauchet ${ }^{1,2,3}$ | Amandine Dufour4 | Rosa Cossart1,2,3 | \\ Constance Hammond1,2,3,5
}

\author{
${ }^{1}$ INSERM U901, Marseille, France \\ ${ }^{2}$ Aix-Marseille University, UMR 901, \\ Marseille, France \\ ${ }^{3}$ INMED, Marseille, France \\ ${ }^{4}$ Neurochlore, INMED, Marseille, France \\ ${ }^{5}$ B\&A Therapeutics, INMED, Marseille, \\ France

\section{Correspondence} \\ Laurie-Anne Gouty-Colomer, Neurochlore, \\ 163 route de Luminy-BP13, 13273 Mar- \\ seille cedex9, France. \\ Email: gouty-colomer@neurochlore.fr \\ Present address \\ Laurie-Anne Gouty-Colomer, Neurochlore, \\ INMED, Marseille 13009, France
}

\begin{abstract}
The neuronal population of the subthalamic nucleus (STN) has the ability to prolong incoming cortical excitation. This could result from intra-STN feedback excitation. The combination of inducible genetic fate mapping techniques with in vitro targeted patch-clamp recordings, allowed identifying a new type of STN neurons that possess a highly collateralized intrinsic axon. The time window of birth dates was found to be narrow (E10.5-E14.5) with very few STN neurons born at E10.5 or E14.5. The fate mapped E11.5-12.5 STN neuronal population included $20 \%$ of neurons with profuse axonal branching inside the nucleus and a dendritic arbor that differed from that of STN neurons without local axon collaterals. They had intrinsic electrophysiological properties and in particular, the ability to generate plateau potentials, similar to that of STN neurons without local axon collaterals and more generally to that of classically described STN neurons. This suggests that a subpopulation of STN neurons forms a local glutamatergic network, which together with plateau potentials, allow amplification of hyperdirect cortical inputs and synchronization of the STN neuronal population.
\end{abstract}

\section{KEYWORDS}

axon collaterals, basal ganglia, calcium binding proteins, inducible genetic fate mapping, patchclamp, subthalamic nucleus, RRID: AB_2650496, RRID: AB_10000340, RRID: AB_10000342, RRID: AB_2336933, RRID: AB_2337249, RRID: AB_2313584

\section{1 | INTRODUCTION}

The ability of brief stimuli to trigger prolonged neuronal activity is common to brain areas related to short-term memory and motor responses. Subthalamic nucleus (STN) neuronal population, in the basal ganglia, has this ability to prolong incoming cortical signals. Brief stimulation of STN neurons or of their cortical afferents evoke long-lasting complex excitatory postsynaptic currents or potentials, (EPSCs, EPSPs) in their target neurons of the external and internal globus pallidus (GPe, GPi) and substantia nigra pars reticulata and compacta (SNr, SNc) (Ammari, Bioulac, Garcia, \& Hammond, 2011; Ammari, Lopez, Bioulac, Garcia, \& Hammond, 2010; Shen \& Johnson, 2006). More importantly, these complex EPSCs or EPSPs reverberate in target STN neurons in a mouse model of Parkinson's disease, that is, in the absence of dopaminergic innervation (Ammari et al., 2011).

Bistable membrane properties and network feedback excitation are both suitable mechanisms to sustain persistent responses. STN neurons exhibit membrane bistability as they generate spontaneous bursting activity and plateau potentials as a result of a cascade of voltage- and calcium-sensitive intrinsic membrane currents (Beurrier, Congar, Bioulac, \& Hammond, 1999; Kass \& Mintz, 2006). Reverberation implies that some neurons in the network feed excitation back onto the others (Koulakov, Raghavachari, Kepecs, \& Lisman, 2002). However, positive feedback excitation in the STN has yet to be supported by the presence of recurrent axon collaterals with terminal boutons inside the STN. Several arguments are in favor of the existence of feedback excitation between rodent or cat STN neurons: (a) Morphological studies identified axon collaterals inside the STN from intracellularly HRP-labeled STN neurons with very few (Chang, Kita, \& Kitai, 1984) or without (Ammari et al., 2010; Iwahori, 1978; Kita, Chang, \& Kitai, 1983) clear terminal boutons; (b) STN-evoked complex EPSCs are polysynaptic, similar in all STN target neurons and depend on the presence of the STN in the slice but not of other glutamatergic afferent neurons such as cortical and pedunculopontine neurons (Ammari et al., 2010). 
We hypothesized that early-born STN neurons would be good candidates for giving off axon collaterals inside the STN based on studies of early-generated hippocampal CA3 neurons which develop into functional hub cells (Marissal et al., 2012; Picardo et al., 2011). To address this issue, we used inducible genetic fate mapping approaches and in vitro targeted patch-clamp recordings to determine the morpho-physiological features of fate mapped STN neurons. During development, glutamatergic neurons transiently express the transcription factor Neurogenin-2 (Ngn2) as they are becoming postmitotic. We have fate mapped glutamatergic neuron precursors using CreER, a tamoxifen-induced cre recombinase whose expression was placed under the control of the Ngn2 loci. Glutamatergic progenitors express Ngn2 in accordance with their exit from mitosis, hence the timing of Ngn2 in a neuronal population provides a precise proxy indicator of their birthdate (Britz et al., 2006). We show that at least $20 \%$ of STN neurons born at embryonic days E11.5-12.5 displayed profuse axon collaterals with endings inside the STN suggesting the existence of a feedforward excitatory network inside the STN.

\section{2 | MATERIALS AND METHODS}

\section{$2.1 \mid$ Animals and fate mapping}

All animal use protocols were performed under the guidelines of the French National Ethic Comity for Sciences and Health report, in agreement with the European Community Directive 86/609/EEC. All efforts were made to minimize pain and suffering and to reduce the number of animals used. To detect the date of birth of glutamatergic neurons of the STN (Rinvik and Ottersen 1993), we used the fate mapping strategy by taking advantage that glutamatergic neurons transiently express the transcription factor Ngn2 during development as they are becoming postmitotic. Eight- to ten-week-old, Swiss females were crossed with Ngn2CreER ${ }^{\mathrm{WT} / \mathrm{Tg}} / \mathrm{Al} 14 \mathrm{Tomato}^{\mathrm{Tg} / \mathrm{Tg}}$ males expressing the inducible CreER recombinase under the endogenous Ngn2 promotor and the fluorescent reporter TdTomato (Al14, Madisen et al., 2010). We fed by gavaging (Feeding Needles, Fine Science Tools, Foster City, CA) pregnant females at embryonic days E10.5-16.5 post vaginal plug, with a tamoxifen solution ( $2 \mathrm{mg}$ tamoxifen solution per $30 \mathrm{~g}$ body weight prepared at $10 \mathrm{mg} / \mathrm{mL}$ in corn oil, Sigma, St Louis, MO). For postnatal neuronal birth dates, we treated pups with an intraperitoneal injection of tamoxifen ( $0.33 \mathrm{mg} / \mathrm{g}$ body weight) at postnatal days (P) P1 and P7. Recombination of the reporter allele was achieved within $24 \mathrm{hr}$ upon administration of tamoxifen, therefore, temporally labeling glutamatergic cells as they become postmitotic and providing a proxy indicator of their birthdate.

\subsection{Cell counting and immunocytochemistry}

We anesthetized 8-week-old Ngn2CreER/Al14 mice (force feeding at E10.5-E16.5 or intraperitoneal injection at P1 or P7) $(n=27)$ with a ketamine (100 mg mL ${ }^{-1}$ ) and xylazine $\left(1 \mathrm{mg} \mathrm{mL}^{-1}\right)$ solution $(10 \mathrm{~mL}$ $\mathrm{kg}^{-1}$ i.p.) and perfused them transcardially with $4 \%$ paraformaldehyde in PB $\left(1 \mathrm{~mL} \mathrm{~g}^{-1}\right)$. Brains were postfixed overnight in fixative and then washed in PBS. We performed sagittal brain sections $(70 \mu \mathrm{m})$ to count the number of Td-Tomato-positive neurons in the whole STN of each side. Brain sections from force feeding time E12.5 mice were routinely processed for immunofluorescence to calcium binding proteins. Briefly, after preincubation in 5\% normal donkey serum, sections were first incubated overnight at room temperature under constant shaking in a solution (PBS plus 0.3\% Trion-X100, PBST) containing goat-anti Parvalbumin (1/2000, PVG-213, Swant ${ }^{\circledR}$, Marly, Switzerland, www. swant.com, RRID: AB_2650496), rabbit anti-Calbindin D-28K (1/2000, Swant ${ }^{\circledR}, \quad$ CB38, Marly, Switzerland, www.swant.com, RRID: AB_10000340) or goat-anti Calretinin (1/2000, CG1, Swant ${ }^{\circledR}$, Marly, Switzerland, www.swant.com, RRID: AB_10000342). After several washes in PBST, sections were incubated for $2 \mathrm{hr}$ in appropriate secondary antibodies (1/500, Alexa Fluor ${ }^{\circledR} 488$ AffiniPure Donkey AntiGoat IgG, RRID: AB_2336933 or Alexa Fluor ${ }^{\circledR} 488$ AffiniPure Donkey Anti-Rabbit IgG (H+L), RRID:AB_2313584 Jackson Immunoresearch Lab. Inc., West Grove, PA, www.jacksonimmuno.com). Following several washes in PBS, then PB, sections were mounted between slide and coverslip within VECTASHIELD Antifade Mounting Medium with DAPI (Vector Lab., Peterborough, https://vectorlabs.com/) before microscopic examination.

\subsection{Slice preparation and patch-clamp recordings}

We prepared sagittal brain slices at the level of the STN $(250-350 \mu \mathrm{m}$ thick) from tamoxifen-treated juvenile (P10-21) Ngn2CreER/Al14 mice using a Leica VT1200 S (Leica Microsystems, Wetzlar, Germany) vibratome in ice-cold oxygenated solution containing in $\mathrm{mM}: 126$ choline, $2.5 \mathrm{KCl}, 1.25 \mathrm{NaH}_{2} \mathrm{PO}_{4}, 7 \mathrm{MgCl}_{2}, 0.5 \mathrm{CaCl}_{2} ; 26 \mathrm{NaHCO}_{3}, 5$ glucose. Slices were transferred for $1 \mathrm{hr}$ in oxygenated standard ACSF containing (in mM): $126 \mathrm{NaCl}, 3.5 \mathrm{KCl}, 1.25 \mathrm{NaH}_{2} \mathrm{PO}_{4}, 1.3 \mathrm{MgCl}_{2}, 2.0 \mathrm{CaCl}_{2}$, $25 \mathrm{NaHCO}_{3}$, and 10 glucose, $\mathrm{pH}$ 7.4. Borosilicate glass pipettes (4-7 MOhms) were filled with a solution containing (in $\mathrm{mM}$ ): $128.5 \mathrm{~K}$ gluconate, $11.5 \mathrm{KCl}, 1 \mathrm{CaCl}_{2}$, 10 EGTA, 10 HEPES, $2.5 \mathrm{Mg}$-ATP, 0.3 Na-GTP and 0.5\% Neurobiotin, pH 7.3, 285-290 mOsm. No correction for liquid junction potential was applied. We recorded the electrophysiological properties of 115 Td-Tomato-positive STN neurons (from 48 mice) in whole cell configuration using the Digidata 1344A interface and the Multiclamp 200B amplifier (Molecular Devices, Sunnyvale, CA). Data were acquired at $20 \mathrm{KHz}$, filtered at $3 \mathrm{KHz}$, stored and analyzed using the pClamp software (pClamp 10, Molecular Devices, Sunnyvale). Uncompensated access resistance values below $30 \mathrm{MOhms}$ were considered acceptable and results were discarded if they changed by $>20 \%$. We analyzed their passive membrane properties in voltageclamp mode in response to a $10 \mathrm{mV}$ hyperpolarizing voltage step $\left(V_{H}=-60 \mathrm{mV}\right)$. We evoked single action potentials (APs) in response to $5 \mathrm{~ms}$ depolarizing current pulses whose amplitude was minimally sufficient to reach spike threshold (from $\mathrm{Vm}=-80 \mathrm{mV}$ ) and analyzed firing properties in response to intracellular sub- and supra-threshold 200 ms current pulses $(20-300 \mathrm{pA})$ at $0.5 \mathrm{~Hz}$. The maximum number of APs was analyzed as the maximum number of evoked APs independently of the injected current intensity. 

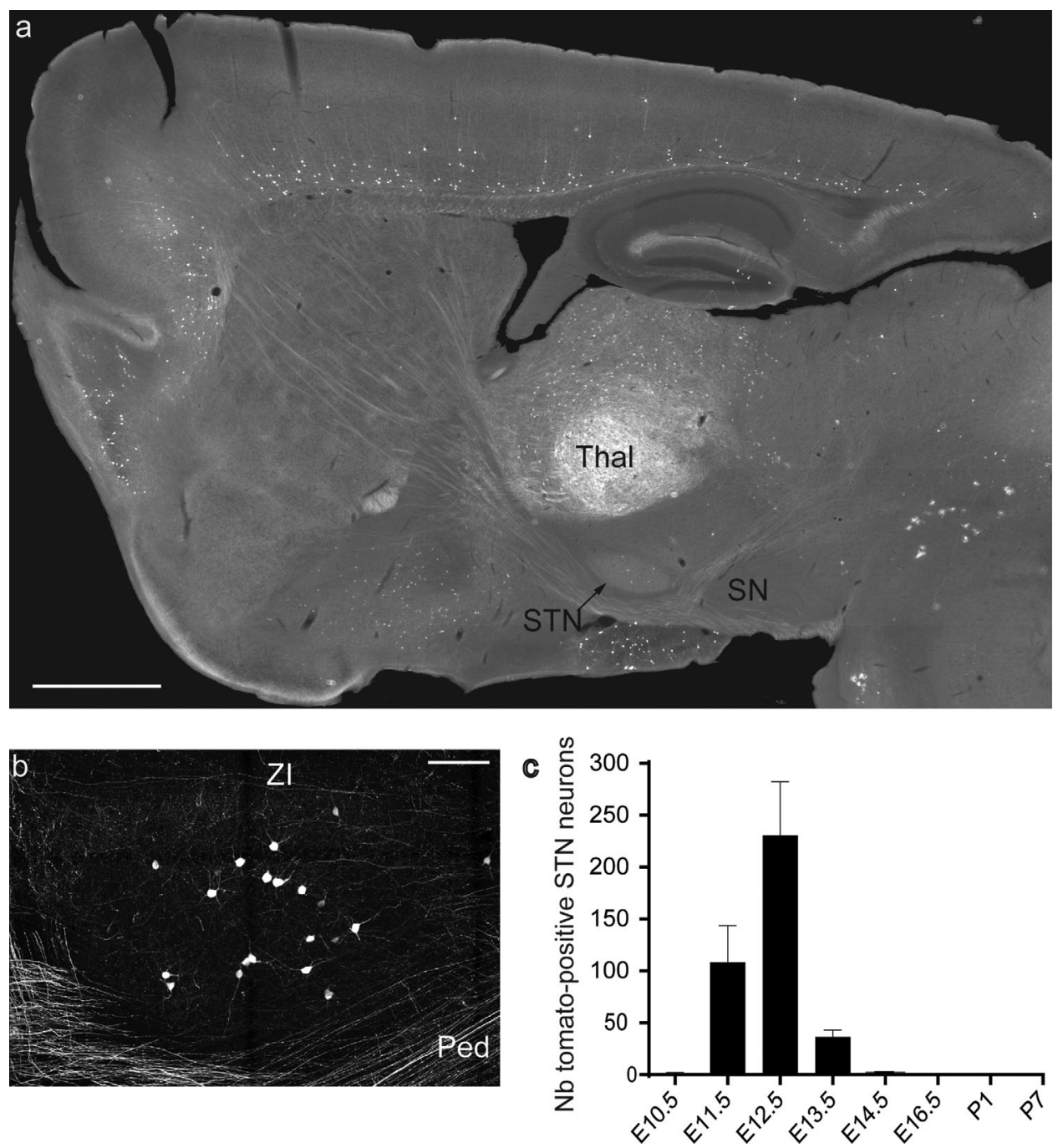

FIGURE 1 Birth dates of STN neurons. (a) Sagittal brain section of Ngn2CreER/AI14 mouse showing the distribution of Td-Tomato neurons (tamoxifen administration at E12.5). (b) Higher magnification of the STN containing numerous Td-Tomato-positive neurons. (c) Diagram showing the number of Td-Tomato-positive STN somata as a function of the embryonic day (E) or postnatal day (P) of tamoxifen administration. Ped: peduncle; SN: substantia nigra; Thal: thalamus; Zl: zona incerta. Scale bars $1 \mathrm{~mm}$ (a), $100 \mu \mathrm{m}$ (b)

\section{4 | Neurobiotin-filled cells morphological analysis}

After the recording session we identified recorded neurons as STN neurons by their location inside the STN and characterized their morphological properties, Slices were fixed overnight at $4^{\circ} \mathrm{C}$ in Antigenfix, rinsed in PBS containing 0.3\% Triton X-100 (PBST) and incubated overnight at room temperature in Alexa Fluor ${ }^{\circledR} 488$-streptavidin (1/1000 in PBST, Jackson Immunoresearch Lab. Inc., West Grove, PA, www.jacksonimmuno.com, RRID: AB_2337249). We performed post-hoc analysis using a confocal microscope (Leica SP5X, Wetzlar, Germany) and reconstructed $96 \mathrm{Td}$-Tomato positive STN neurons with a computerassisted system (Neurolucida software, v10, MBF Bioscience, Williston, VT) and edited with Fiji program (the open-source platform Fiji) (Schindelin et al., 2012). We included 39 out of the 96 labeled STN neurons in the morphometric analysis. The criteria were the good quality of intracellular labeling and the presence of an axon of a length $>300 \mu \mathrm{m}$. We chose this minimal length value of $300 \mu \mathrm{m}$ based on the maximal length of the parent axon before its first bifurcation. Local axon collaterals of STN neurons, when present, bifurcated 2-15 times before $300 \mu \mathrm{m}$. In the quantification of local axonal terminals, we did not take into account axonal varicosities.

\section{5 | Statistical analysis}

We used Prism 6 (GraphPad Software Inc., La Jolla, CA) for statistical analysis. We established significance of all data using Mann-Whitney test except for Sholl analyses (Kolmogorov-Smirnov test). Proportions were compared with Chi-square (and Fisher's exact test). Data were expressed as mean \pm SEM. Significance threshold was set at $p \leq .05$ for all statistical comparisons.

\section{3 | RESULTS}

We first identified the birth dates of STN neurons in mice. The plot of the number of Td-Tomato-positive STN neurons (having undergone the reporter allele recombination) present in each STN (Figure 1a,b) 

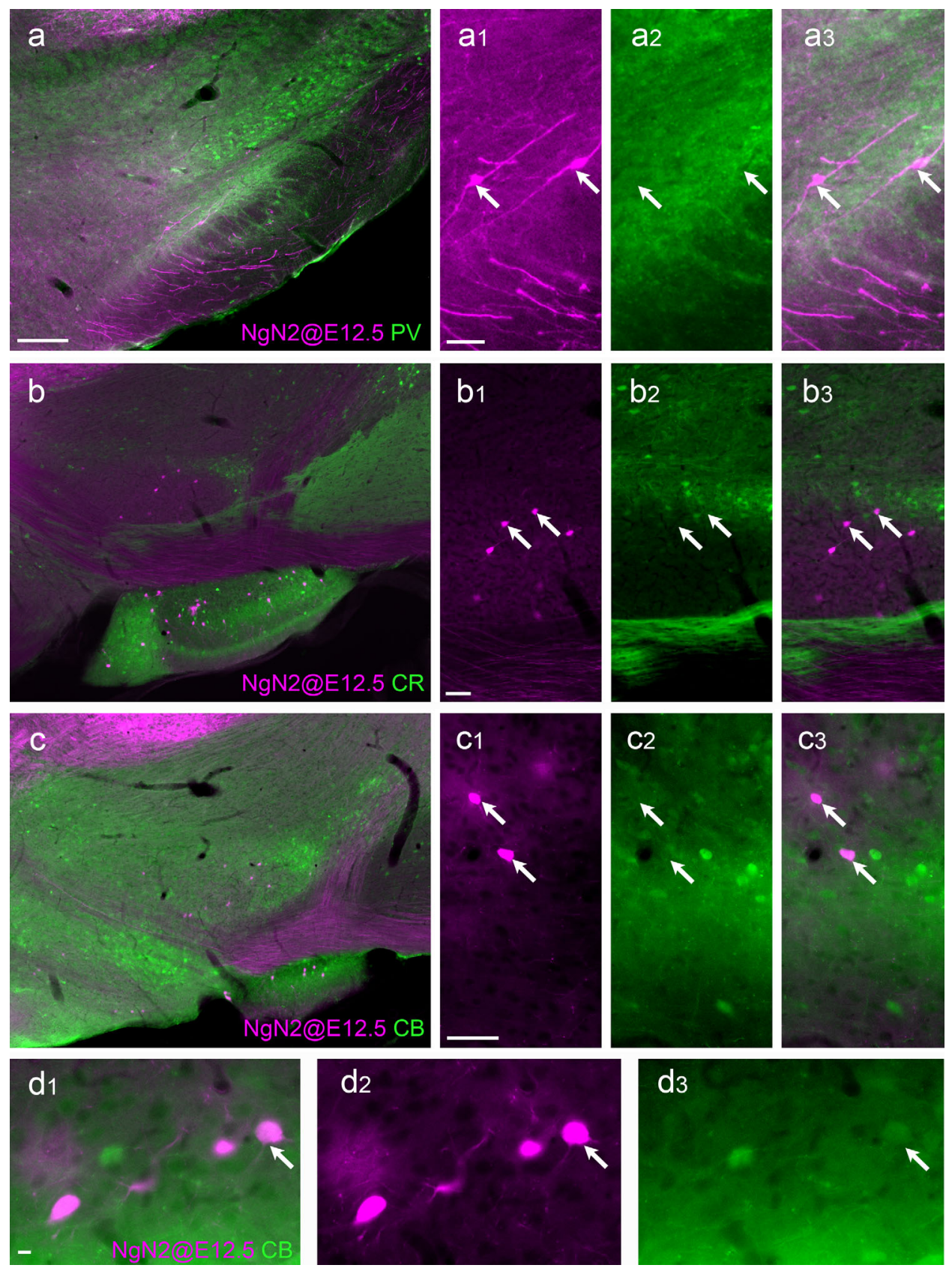

FIGURE 2 Expression of calcium-binding proteins in E12.5 fate mapped STN neurons. E12.5 fate mapped neurons within the STN do not express immunofluorescence neither for parvalbumin (PV, a) nor for calretinin (CR, b). Most of the E12.5 fate mapped neurons do not show immunofluorescence for calbindin (CB, c, d). Arrow in d1-3 shows co-expression of Td-Tomato and CB. Arrows in a1-3, b1-3, and c1-3 show Td-Tomato positive neurons without calcium-binding protein expression. Scale bars $100 \mu \mathrm{m}$ (a, b, c); 50 $\mu \mathrm{m}$ (a1-3, b1-3, c1-3); $10 \mu \mathrm{m}(\mathrm{d} 1-3)$ [Color figure can be viewed at wileyonlinelibrary.com]

( $n=27$ mice) against their date of birth (date of tamoxifen administration) (Figure 1c) showed that the majority of glutamatergic STN neurons was generated between E10.5 and E14.5. Almost 99\% ( $n=1573$ ) 1583) were generated during the E11.5-E13.5 period with a clear peak at E12.5 ( $n=922 / 1583 ; 58 \%$ ). We did not identify STN glutamatergic neurons generated at E16.5, P1 or P7. This shows that STN neurons arrive early in the STN and within a narrow time window in comparison to rodent excitatory cortical neurons (Angevine \& Sidman, 1961; Marissal et al., 2012). We identified a cytogenetic gradient in the developing
STN with neurons born at E10.5-E11.5 occupying the more rostral and dorsolateral regions of the STN (not shown). Of the 204 Td-Tomatopositive STN neurons from adult Ngn2CreER/Al14 mice force-fed at E12.5 $(n=4)$ none expressed parvalbumin $(0 / 82)$ (Figure 2a) or calretinin $(0 / 25)$ (Figure $2 b$ ) and only two expressed calbindin ( $n=2 / 97$ ) (Figure $2 c, d)$. This showed that E12.5 fate mapped STN neurons did not express a particular calcium-binding protein. In contrast, we clearly identified Td-Tomato-negative STN neurons that were immunopositive for parvalbumin or calretinin. 
a
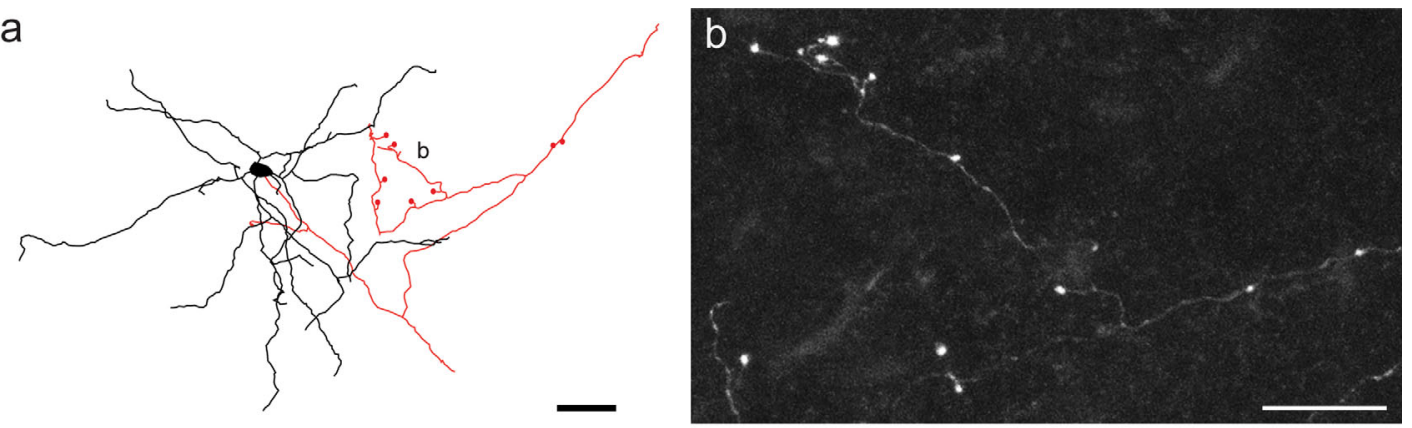

C

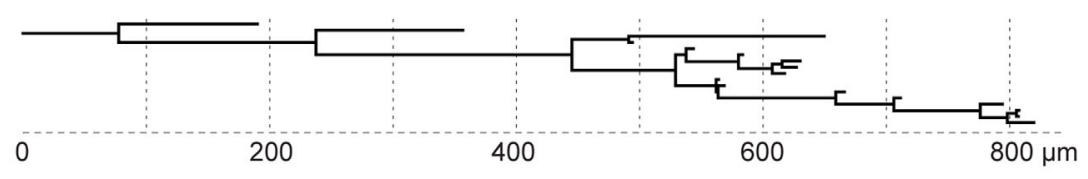

d
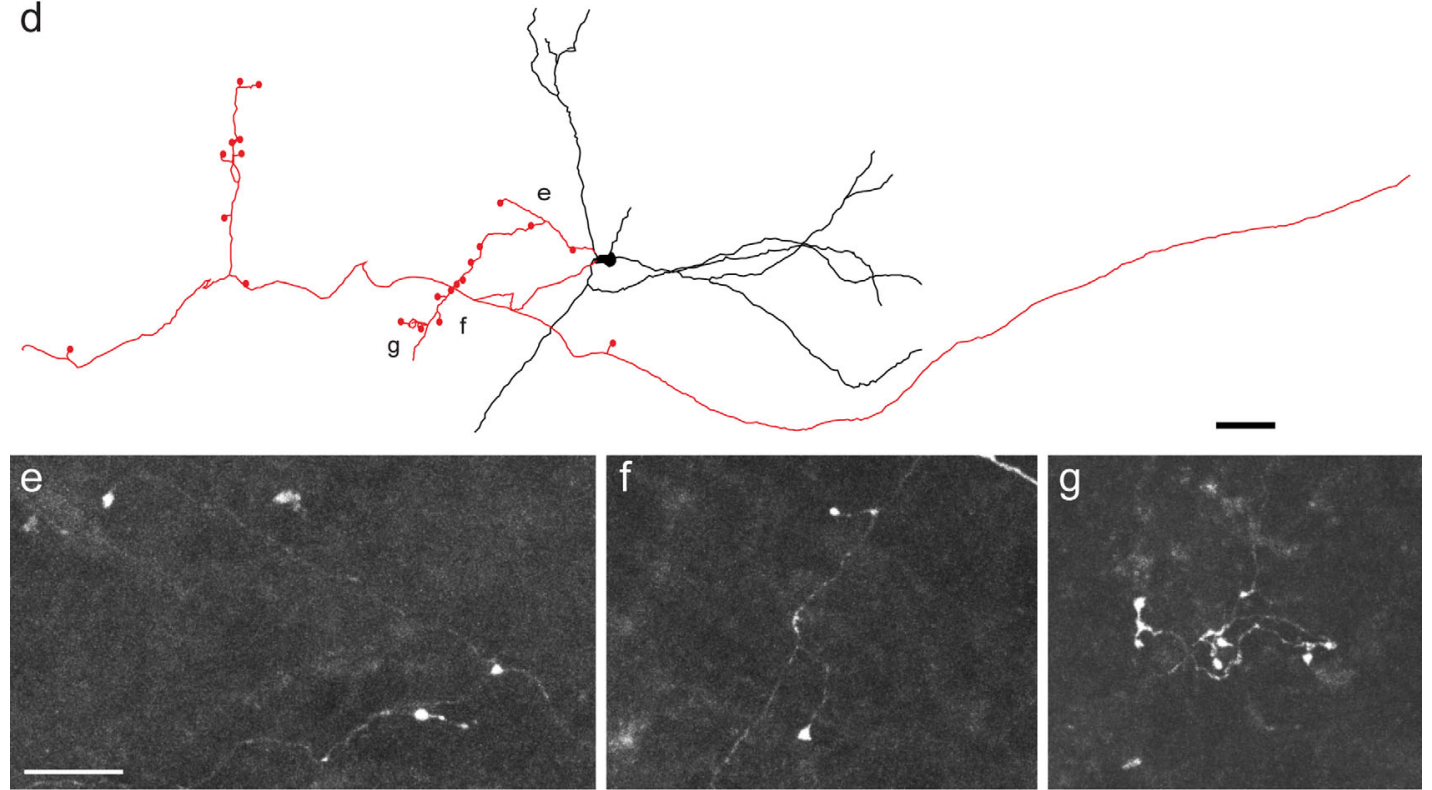

$\mathrm{h}$

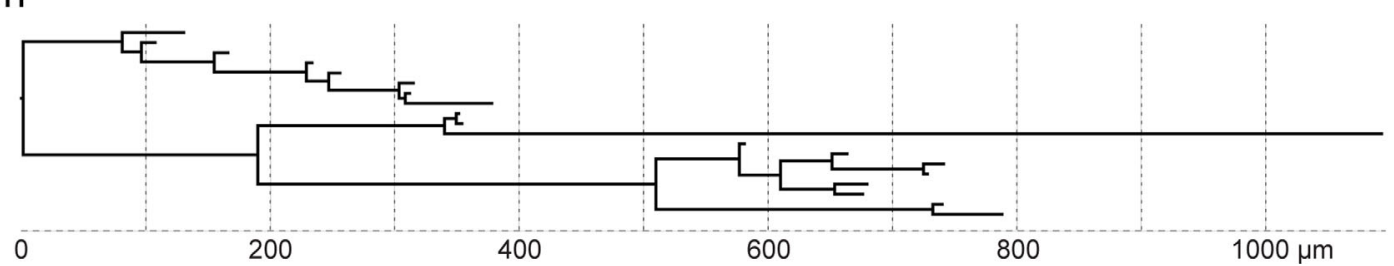

FIGURE 3 Examples of E11.5-12.5 fate mapped STN neurons with local axon collaterals. (a, d) Neurolucida reconstruction of two neurons with local axon collaterals. The dendritic trees are in black and the axonal arbors in red. Confocal photomicrographs of the axon collaterals and terminal-like boutons at places indicated in neuron a (b) or neuron d (e-g). Axograms corresponding to neuron a (c) or neuron $d(h)$. Scale bars: $50 \mu \mathrm{m}$ (a, d), $20 \mu \mathrm{m}$ (b) and $10 \mu \mathrm{m}(\mathrm{e}-\mathrm{g})$ [Color figure can be viewed at wileyonlinelibrary.com]

In order to detect whether a subpopulation of fate mapped STN neurons had intra-STN axon collaterals, we labeled E11.5-12.5 Td-Tomato-positive STN neurons $(n=115)$ with neurobiotin during patch-clamp recordings. We discarded neurons that were not clearly identifiable $(n=19)$, and those which had no axon or an axon shorter than $300 \mu \mathrm{m}(n=57)$ (see methods). The remaining 39 STN neurons had the classically described, simple, bifurcated, or trifurcated, long range projecting axons running up to the substantia nigra and/or globus pallidus. Among them, nearly $20 \%(n=7 / 39)$ had also intra-STN axon collaterals (Figure 3a,d). The number of axonal nodes of local axon collaterals ranged from 4 to $19(11 \pm 2$ ) (Figure $3 c, h$ ). The number of intra-nucleus end-terminals ranged from 5 to 19 with the vast majority $(n=52 / 80 ; 65 \%)$ resembling synaptic terminal boutons (Figure $3 \mathrm{~b}, \mathrm{e}-\mathrm{g}$ ). STN local axon collaterals had a total length of $850.6 \pm 124.1 \mu \mathrm{m}$ per neuron and occupied a volume between 94263 and $2185940 \mu \mathrm{m}^{3}$. Thus, we identified a sub-population of STN neurons endowed with intra-nucleus axon collaterals showing a rather large axonal arborization (Table 1). 
TABLE 1 Morphological and electrophysiological characteristics of E11.5-12.5 fate mapped STN neurons with or without axon collaterals

\begin{tabular}{|c|c|c|c|c|}
\hline Morphological properties & & $\begin{array}{l}\text { With axon collaterals } \\
n=7\end{array}$ & $\begin{array}{l}\text { Without axon collaterals } \\
n=32\end{array}$ & $p$ value \\
\hline Cell body area & $\left(\mu \mathrm{m}^{2}\right)$ & $143.1 \pm 19.7$ & $161.7 \pm 11.7$ & .69 \\
\hline \multirow[t]{4}{*}{ Axon collaterals } & $\mathrm{Nb}$ axonal nodes & $11 \pm 2$ & & \\
\hline & $\mathrm{Nb}$ intra-nucleus end-terminals & $14.4 \pm 2.3$ & & \\
\hline & Axon collateral length $(\mu \mathrm{m})$ & $850.6 \pm 124.1$ & & \\
\hline & Axon collateral volume $\left(\mu \mathrm{m}^{3}\right)$ & $698594 \pm 292927$ & & \\
\hline \multirow[t]{8}{*}{ Dendritic tree } & $\mathrm{Nb}$ dendritic trunks & $3.8 \pm 0.5$ & $4.0 \pm 0.3$ & .91 \\
\hline & $\mathrm{Nb}$ dendritic ends & $18.8 \pm 3.1$ & $14.0 \pm 0.7$ & .14 \\
\hline & Dendritic end/trunk & $5.0 \pm 0.6$ & $3.8 \pm 0.2^{*}$ & .04 \\
\hline & Total dendritic length $(\mu \mathrm{m})$ & $2503 \pm 197$ & $2090 \pm 121$ & .10 \\
\hline & Maximal nb intersections & $13.6 \pm 1.0$ & $11.5 \pm 0.5$ & .05 \\
\hline & Dendritic volume $\left(\mu \mathrm{m}^{3}\right)$ & $21.010^{6} \pm 5.310^{6}$ & $18.810^{6} \pm 2.910^{6}$ & .65 \\
\hline & Critical radius $(\mu \mathrm{m})$ & $135.7 \pm 20.6$ & $127.0 \pm 16.8$ & .37 \\
\hline & Ending radius $(\mu \mathrm{m})$ & $292.3 \pm 29.0$ & $329.9 \pm 14.7$ & .28 \\
\hline Electrophysiolo-gical properties & & $n=5$ & $n=18$ & \\
\hline \multirow[t]{2}{*}{ Membrane } & Resistance (M $\Omega$ ) & $285.6 \pm 39.0$ & $327.9 \pm 31.8$ & .65 \\
\hline & Capacitance (pF) & $29.0 \pm 2.0$ & $29.6 \pm 1.5$ & .60 \\
\hline \multirow[t]{7}{*}{ Action potential } & Rheobase (pA) & $430 \pm 12$ & $415 \pm 36$ & .81 \\
\hline & Threshold (mV) & $-34.6 \pm 2.5$ & $-38.5 \pm 1.2$ & .16 \\
\hline & Peak amplitude (mV) & $51.0 \pm 6.2$ & $60.7 \pm 2.4$ & .95 \\
\hline & Half-width (ms) & $1.09 \pm 0.14$ & $0.87 \pm 0.11$ & .10 \\
\hline & Rise time (ms) & $0.55 \pm 0.06$ & $0.45 \pm 0.05$ & .08 \\
\hline & Decay time (ms) & $0.84 \pm 0.13$ & $0.67 \pm 0.12$ & .07 \\
\hline & AHP amplitude (mV) & $23.3 \pm 3.5$ & $20.6 \pm 1.3$ & .33 \\
\hline \multirow[t]{2}{*}{ Firing } & Maximal nb of AP & $8.8 \pm 3.6$ & $15.8 \pm 2.2$ & .19 \\
\hline & Delay to first AP (ms) & $9.2 \pm 0.7$ & $9.9 \pm 1.2$ & .98 \\
\hline
\end{tabular}

To better characterize the morphological properties of the two subpopulations of STN neurons, those with axon collaterals and those without, we analyzed their somato-dendritic arbors (Figure 4a-d and Table 1). The two groups had similar mean soma size, total dendritic length and number of dendritic trunks (Table 1). In contrast, the Sholl analysis showed different distributions of dendrite intersections in the two populations of neurons with the STN neurons with axon collaterals having more intersections $(p \leq .0001)$ (Figure $4 \mathrm{e})$ though the value of the critical radius (radius at which there is the maximum number of dendritic intersections) was similar (Table 1). Their ratio of dendritic ends per dendritic trunk was also higher $(p \leq .05)$ (Table 1, Figure 4f). The above results suggested that STN neurons that give off intranucleus axon collaterals have larger dendritic arbors. These collateralized STN neurons did not show preferential distribution inside the STN, either rostro-caudally or latero-medially (Figure 4g).

The two subpopulations of E11.5-12.5 STN neurons had similar mean membrane resistance and capacitance (Table 1). Mean properties of single action potentials (rheobase, threshold, peak amplitude, half-width duration, and AHP amplitude) and evoked or spontaneous firing were also similar for the two groups (Figure $5 a-c$ and Table 1). The proportion of fate mapped STN neurons exhibiting plateau potentials (Figure $5 b, e)$ was the same in both groups (2/5 and $6 / 18$, respectively; $p=.39$ ). Overall, the two subpopulations of fate mapped STN neurons displayed similar electrophysiological characteristics and similar as those already described for juvenile STN neurons (Beurrier, Bioulac, \& Hammond, 2000; Beurrier et al., 1999; Bevan \& Wilson, 1999).

\section{4 | DISCUSSION}

We showed that nearly $20 \%$ of identified and reconstructed fate mapped STN neurons displayed profuse axonal branching with endings inside the STN. In addition, collateralized neurons displayed a more profuse dendritic arbor compared to that of neurons without axon 


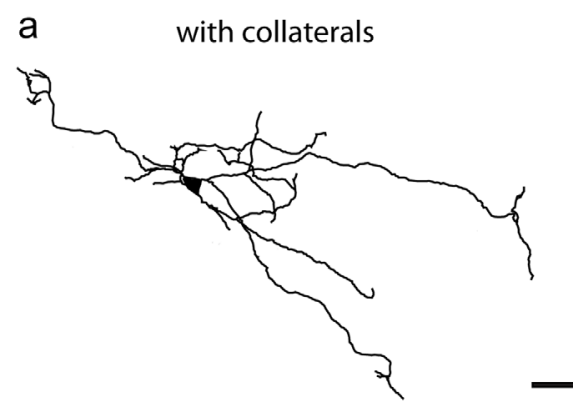

b

without collaterals

C
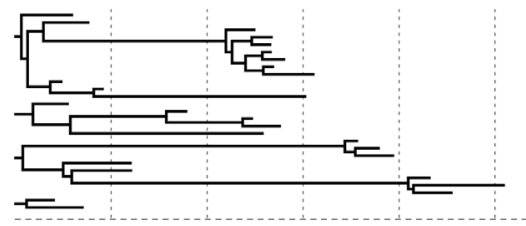

0

200

$400 \mu \mathrm{m}$

e

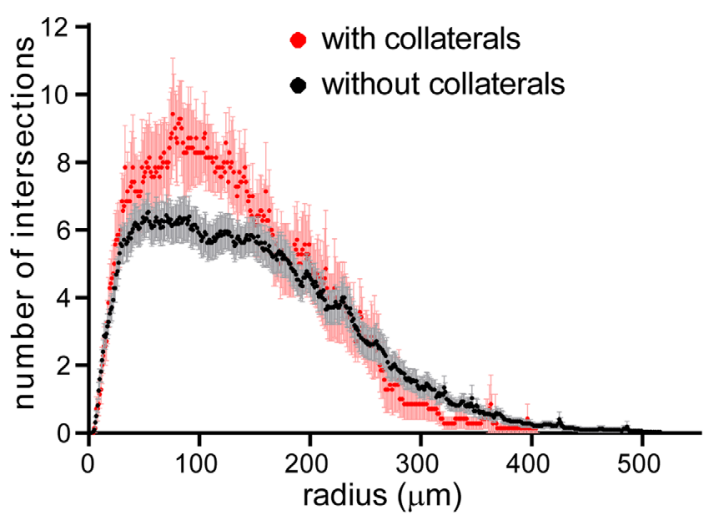

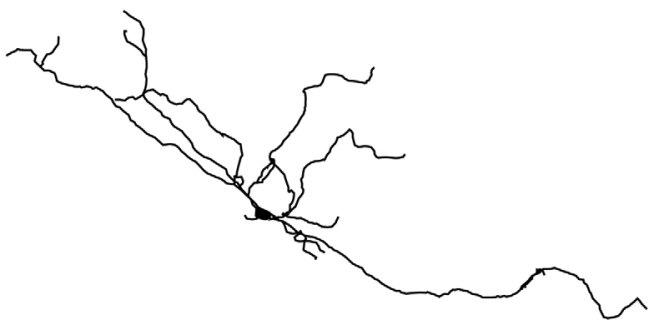

d

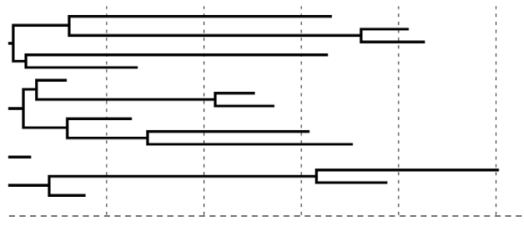

0

f

g
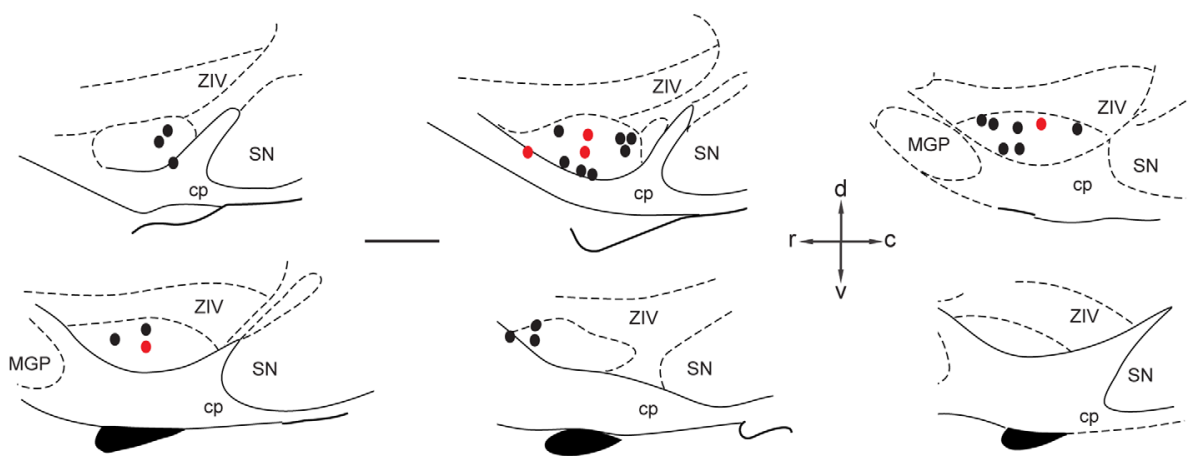

FIGURE 4 Dendritic trees of E11.5-12.5 fate mapped STN neurons. Representative dendritic trees of neurons, with (a) or without (b) local axon collaterals. (c, d) Corresponding dendrograms. Sholl analysis ( $1 \mu \mathrm{m}$ bin) of the dendritic trees of the two types of STN neurons (c) and diagram showing the ratio of the number of dendritic ends versus trunks (d). Scale bar $50 \mu \mathrm{m}$. (g) Rostro-caudal and medio-lateral distribution of E11.5-12.5 fate mapped STN neurons with (red) or without (black) local axon collaterals. The sagittal planes are from left to right and from top to bottom at lateral 1.32, 1.44, 1.56, 1.68, 1.80, $1.92 \mathrm{~mm}$ (Franklin KBJ mouse brain atlas, 2001). Scale bar: $500 \mu \mathrm{m}$ [Color figure can be viewed at wileyonlinelibrary.com]

collaterals, showing that the STN neuronal population is not morphologically homogenous. In contrast, the two populations of STN neurons had similar intrinsic membrane properties and similar to those previously described in STN neurons. In particular, half of these neurons could generate plateau potentials. These results suggest the existence of a feedforward excitatory network within the STN. Such a network, together with bistable membrane properties might amplify incoming cortical signals and synchronize STN neurons resulting in strong activation of basal ganglia output neurons and inhibition of motor thalamo-cortical networks.

\section{1 | Birth dates of mouse STN neurons and cytogenetic gradient in the developing STN}

Interestingly, STN neurons are generated early in embryogenesis and during a limited period of time ( 5 days) in the mouse. In the rat, STN 

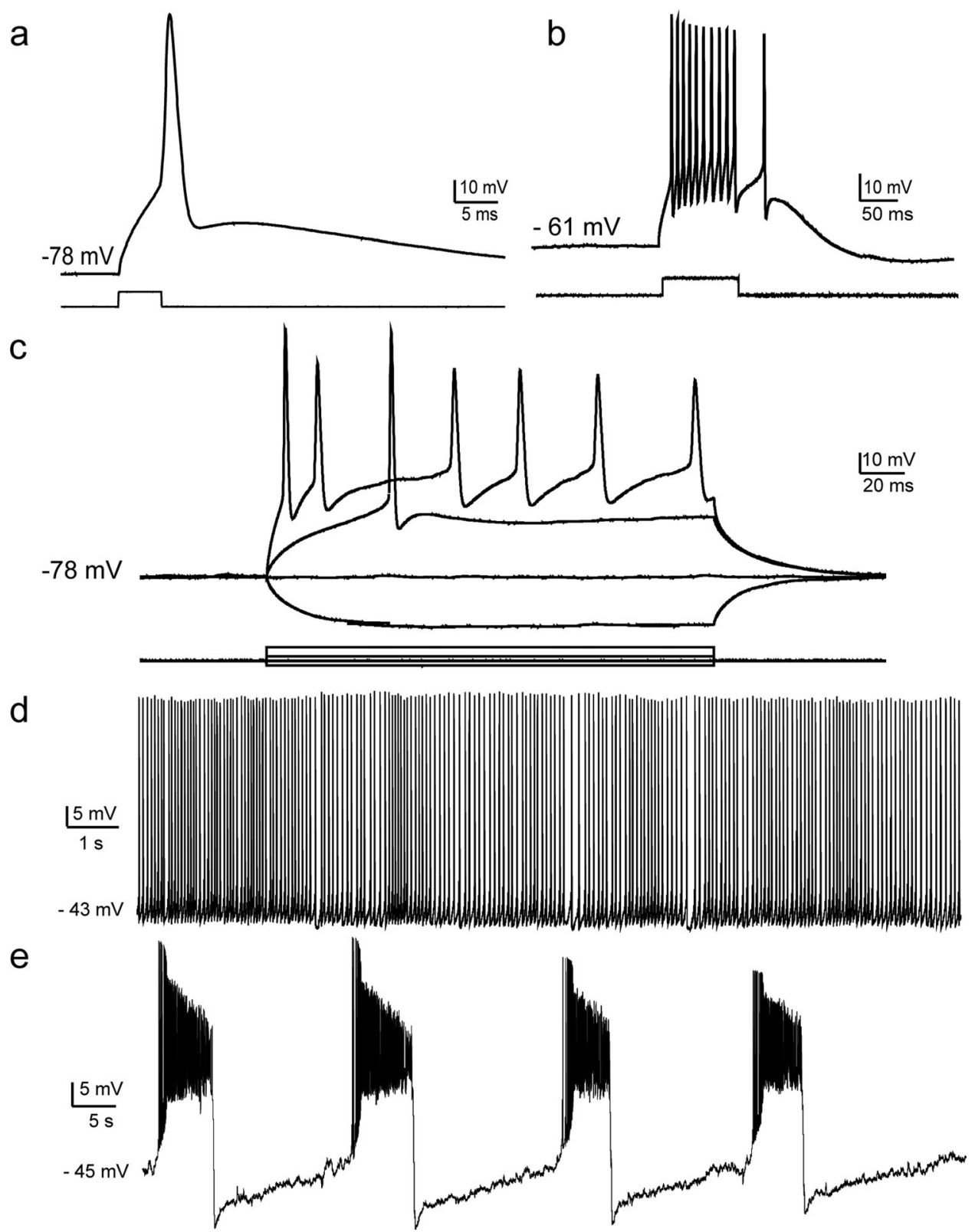

FIGURE 5 Action potential and firing properties of E11.5-12.5 fate mapped STN neurons with local axon collaterals. (a) Single action potential in response to a depolarizing step ( $+400 \mathrm{pA}, 5 \mathrm{~ms}$ ) from $\mathrm{Vm}=-78 \mathrm{mV}$ and (b) spike discharge in response to a depolarizing step $(+100 \mathrm{pA}, 100 \mathrm{~ms})$ from $\mathrm{Vm}=-61 \mathrm{mV}$. (c) Current clamp responses to hyperpolarizing $(-100 \mathrm{pA})$ and depolarizing steps (+100 and +300 $\mathrm{pA}, 200 \mathrm{~ms}$ ) from $\mathrm{Vm}=-78 \mathrm{mV}$. Tonic (d) and bursting (e) spontaneous activities recorded at similar $\mathrm{Vm}$. The five representative traces were obtained from four different neurons

neurons are born between embryonic days 12 and 15 in the ventrocaudal aspect of the diencephalic neuroepithelium, with the bulk of neurons (60\%) born at E14-E15 (Altman \& Bayer, 1979; Marchand, 1987). This is comparable to the birth dates identified in mice in the present study considering the longer duration of embryogenesis in rats (21 days instead of 19). STN neurons originate from the retromammillary region of prosomere four, which occupies the basal plate of the diencephalon (Puelles \& Rubenstein, 2003). After exiting the cell cycle, prospective STN neurons migrate latero-dorsally, crossing over the descending tracts of the developing cerebral peduncle. They migrate over a 2-day period according to an outside-in pattern, with the earliest born neurons occupying the most rostral and dorsolateral regions of the STN, and later neurons residing caudal and ventromedial to earlier neurons (Altman \& Bayer, 1979). The cytogenetic gradient identified in mice in the present study is in agreement with the above results in rats.

\section{2 | Calcium binding proteins}

STN neurons do not all contain the same calcium binding proteins (Augood, Waldvogel, Munkle, Faull, \& Emson, 1999; Hontanilla, Parent, de las Heras, \& Gimenez-Amaya, 1998; Morel, Loup, Magnin, \& Jeanmonod, 2002). If E12.5 STN neurons with local axon collaterals 
expressed a particular calcium binding protein, at least $20 \%$ of them should have been positive for parvalbumin, calretinin or calbindin. The absence of such result suggests that calcium-binding proteins are not specific markers of collateralized STN neurons and prevents their easy identification.

\subsection{Morphological-functional characteristics}

All rodent STN neurons possess long-range axons, which bifurcate or trifurcate before running to internal and/or external pallidal segments (GPi in primates or entopeduncular nucleus (EP) in rodents, GPe in primates or GP in rodents) and SN. The situation is somewhat different in primates where the majority of STN axons have a single branch coursing rostrally to GPe and GPi. Therefore the primate STN neuronal population is not as homogenous as in rodents with regard to their projection sites (Sato, Parent, Levesque, \& Parent, 2000). The present result also seriously challenges the homogeneity of rodent STN neurons since some of them also display profuse local axon collaterals but the others do not. Previous studies in rodents including our own have identified different proportions of STN neurons with short recurrent axon collaterals (Ammari et al., 2010; Chang et al., 1984; Hammond \& Yelnik, 1983; Kita et al., 1983) whereas they have not yet been described in primates (Sato et al., 2000). The difference between the present study and previous ones is the extent of labeling of these collaterals and the identification of putative terminal boutons. Truncation of axon collaterals due to the slicing procedure in the present study may have resulted in underestimation of the number of retrieved boutons.

Previous reports described rat STN neurons as displaying five to eight long, sparsely spined dendrites arborizing mostly along the main axis of the nucleus (Afsharpour, 1985; Hammond \& Yelnik, 1983). Mouse fate mapped STN neurons had a mean of four dendritic trunks (2-9) and their dendrites were also sparsely spined. The ratio of the number of dendritic ends/trunks between collateralized and noncollateralized STN neurons was different suggesting that collateralized STN neurons had a more ramified dendritic tree, as previously reported in the rat (Kita et al., 1983). This was supported by the Sholl analysis showing a higher number of intersections. However, both groups of neurons had similar numbers of dendritic ends and trunks.

All intrinsic membrane properties tested at juvenile stage for fate mapped collateralized STN neurons were similar to that of fate mapped STN neurons without axon collaterals and to that of the juvenile STN neuronal population in general (Beurrier et al., 2000; Beurrier et al., 1999; Bevan, Magill, Hallworth, Bolam, \& Wilson, 2002). Therefore, STN neurons with local axon collaterals are yet impossible to identify from their electrophysiological characteristics during patch-clamp recordings. Only post-hoc morphological studies can do so.

\section{4 | Functional consequences}

If local STN axon collaterals contact dendrites of other STN neurons (Chang et al., 1984), they are good candidates for sustaining a polysynaptic activity in the STN (Gillies \& Willshaw, 1998). Indeed, a single stimulation of cortical afferents to the STN can evoke STN synchronization and widespread long lasting polysynaptic excitations in target nuclei and the mechanism of amplification resides in the STN itself (Magill, Sharott, Bevan, Brown, \& Bolam, 2004); Ammari et al., 2010; Shen \& Johnson, 2006). We propose that it combines the effect of the STN polysynaptic excitatory network and that of voltage dependent depolarizing currents of the STN neuronal membrane. Such amplification would underlie the broad suppressive effect (inhibition of action) implemented by the cortico-STN pathway, which then via GPi and SNr suppresses thalamocortical drive (reviewed in Wessel \& Aron, 2017).

\section{ACKNOWLEDGMENTS}

We thank Pr David Anderson for providing the Ngn2 ${ }^{\text {CreERTM }}$ mouse. We thank Laurène Save for her help with postnatal tamoxifen injection experiments, and the INMED Animal Facility headed by Ms. S. Pellegrino Corby. This work was supported by ANR EbGluNet (to $\mathrm{RC}, \mathrm{CH}), \mathrm{B} \& \mathrm{~A}$ therapeutics $(\mathrm{CH})$ and Neurochlore (LAGC, AD).

\section{CONFLICT OF INTEREST}

The authors declare no conflict of interest.

\section{AUTHOR CONTRIBUTIONS}

Study concept and design: $\mathrm{CH}$, RC. Data acquisition: LAGC, FM, AB, $A D$. Analysis and interpretation of data: LAGC, $A B, A D, C H$. Drafting of the manuscript: LAGC, $C H$. Statistical analysis: LAGC, $A B, A D$. Final approval: $\mathrm{CH}$, LAGC, $\mathrm{AB}, \mathrm{AD}, \mathrm{FM}$. Obtained funding: $\mathrm{RC}, \mathrm{CH}$. Technical and material support: FM, CLP.

\section{ORCID}

Laurie-Anne Gouty-Colomer (iD) http://orcid.org/0000-0002-5545-059X

\section{REFERENCES}

Afsharpour, S. (1985). Light microscopic analysis of Golgi-impregnated rat subthalamic neurons. Journal of Comparative Neurology, 236(1), 1-13.

Altman, J., \& Bayer, S. A. (1979). Development of the diencephalon in the rat. IV. Quantitative study of the time of origin of neurons and the internuclear chronological gradients in the thalamus. Journal of Comparative Neurology, 188(3), 455-471. https://doi.org/10.1002/ cne. 901880308

Ammari, R., Bioulac, B., Garcia, L., \& Hammond, C. (2011). The subthalamic nucleus becomes a generator of bursts in the dopamine-depleted state. Its high frequency stimulation dramatically weakens transmission to the globus pallidus. Frontiers in Systems Neuroscience, 5, 43. https://doi.org/10.3389/fnsys.2011.00043 [doi]

Ammari, R., Lopez, C., Bioulac, B., Garcia, L., \& Hammond, C. (2010). Subthalamic nucleus evokes similar long lasting glutamatergic excitations in pallidal, entopeduncular and nigral neurons in the basal ganglia slice. Neuroscience, 166(3), 808-818. https://doi.org/S0306-4522(10) 00012-6 [pii];10.1016/j.neuroscience.2010.01.011 [doi]

Angevine, J. B., Jr., \& Sidman, R. L. (1961). Autoradiographic study of cell migration during histogenesis of cerebral cortex in the mouse. Nature, 192, 766-768

Augood, S. J., Waldvogel, H. J., Munkle, M. C., Faull, R. L., \& Emson, P. C. (1999). Localization of calcium-binding proteins and GABA transporter (GAT-1) messenger RNA in the human subthalamic nucleus. Neuroscience, 88(2), 521-534. https://doi.org/S0306452298002267 [pii] 
Beurrier, C., Bioulac, B., \& Hammond, C. (2000). Slowly inactivating sodium current (INap) underlies single-spike activity in rat subthalamic neurons. Journal of Neurophysiology, 83(4), 1951-1957.

Beurrier, C., Congar, P., Bioulac, B., \& Hammond, C. (1999). Subthalamic nucleus neurons switch from single-spike activity to burst- firing mode. Journal of Neuroscience, 19(2), 599-609.

Bevan, M. D., Magill, P. J., Hallworth, N. E., Bolam, J. P., \& Wilson, C. J. (2002). Regulation of the timing and pattern of action potential generation in rat subthalamic neurons in vitro by GABA-A IPSPs. Journal of Neurophysiology, 87(3), 1348-1362.

Bevan, M. D., \& Wilson, C. J. (1999). Mechanisms underlying spontaneous oscillation and rhythmic firing in rat subthalamic neurons. Journal of Neuroscience, 19(17), 7617-7628.

Britz, O., Mattar, P., Nguyen, L., Langevin, L. M., Zimmer, C., Alam, S., ... Schuurmans, C. (2006). A role for proneural genes in the maturation of cortical progenitor cells. Cerebral Cortex, 16 Suppl 1, i138-i151. https://doi.org/16/suppl_1/i138 [pii];10.1093/cercor/bhj168 [doi]

Chang, H. T., Kita, H., \& Kitai, S. T. (1984). The ultrastructural morphology of the subthalamic-nigral axon terminals intracellularly labeled with horseradish peroxidase. Brain Research, 299(1), 182-185.

Gillies, A. J., \& Willshaw, D. J. (1998). A massively connected subthalamic nucleus leads to the generation of widespread pulses. Proc Biol Sci., 265(1410), 2101-2109.

Hammond, C., \& Yelnik, J. (1983). Intracellular labelling of rat subthalamic neurones with horseradish peroxidase: Computer analysis of dendrites and characterization of axon arborization. Neuroscience, 8(4), 781-790.

Hontanilla, B., Parent, A., de las Heras, S., \& Gimenez-Amaya, J. M. (1998). Distribution of calbindin D-28k and parvalbumin neurons and fibers in the rat basal ganglia. Brain Research Bulletin, 47(2), 107-116.

Iwahori, N. (1978). A Golgi study on the subthalamic nucleus of the cat. Journal of Comparative Neurology, 182(3), 383-397.

Kass, J. I., \& Mintz, I. M. (2006). Silent plateau potentials, rhythmic bursts, and pacemaker firing: Three patterns of activity that coexist in quadristable subthalamic neurons. Proceedings of the National Academy of Sciences of the United States of America, 103(1), 183188

Kita, H., Chang, H. T., \& Kitai, S. T. (1983). The morphology of intracellularly labeled rat subthalamic neurons: A light microscopic analysis. Journal of Comparative Neurology, 215(3), 245-257. https://doi.org/ 10.1002/cne.902150302 [doi]

Koulakov, A. A., Raghavachari, S., Kepecs, A., \& Lisman, J. E. (2002). Model for a robust neural integrator. Nature Neuroscience, 5(8), 775782. https://doi.org/10.1038/nn893

Madisen, L., Zwingman, T. A., Sunkin, S. M., Oh, S. W., Zariwala, H. A., Gu, H., ... Zeng, H. (2010). A robust and high-throughput Cre reporting and characterization system for the whole mouse brain. Nature Neuroscience, 13(1), 133-140. https://doi.org/10.1038/nn.2467
Magill, P. J., Sharott, A., Bevan, M. D., Brown, P., \& Bolam, J. P. (2004). Synchronous unit activity and local field potentials evoked in the subthalamic nucleus by cortical stimulation. Journal of Neurophysiology, 92(2), 700-714.

Marchand, R. (1987). Histogenesis of the subthalamic nucleus. Neuroscience, 21(1), 183-195.

Marissal, T., Bonifazi, P., Picardo, M. A., Nardou, R., Petit, L. F., Baude, A., ... Cossart, R. (2012). Pioneer glutamatergic cells develop into a morpho-functionally distinct population in the juvenile CA3 hippocampus. Nature Communications, 3, 1316. https://doi.org/ ncomms2318 [pii];10.1038/ncomms2318 [doi]

Morel, A., Loup, F., Magnin, M., \& Jeanmonod, D. (2002). Neurochemical organization of the human basal ganglia: Anatomofunctional territories defined by the distributions of calcium-binding proteins and SMI32. Journal of Comparative Neurology, 443(1), 86-103.

Picardo, M. A., Guigue, P., Bonifazi, P., Batista-Brito, R., Allene, C., Ribas, A., ... Cossart, R. (2011). Pioneer GABA cells comprise a subpopulation of hub neurons in the developing hippocampus. Neuron, 71(4), 695-709. https://doi.org/S0896-6273(11)00546-0 [pii];10.1016/j. neuron.2011.06.018 [doi]

Puelles, L., \& Rubenstein, J. L. (2003). Forebrain gene expression domains and the evolving prosomeric model. Trends in Neurosciences, 26(9), 469-476. https://doi.org/10.1016/s0166-2236(03)00234-0

Rinvik, E., \& Ottersen, O. P. (1993). Terminals of subthalamonigral fibres are enriched with glutamate-like immunoreactivity: An electron microscopic, immunogold analysis in the cat. Journal of Chemical Neuroanatomy, 6(1), 19-30.

Sato, F., Parent, M., Levesque, M., \& Parent, A. (2000). Axonal branching pattern of neurons of the subthalamic nucleus in primates. Journal of Comparative Neurology, 424(1), 142-152.

Schindelin, J., Arganda-Carreras, I., Frise, E., Kaynig, V., Longair, M. Pietzsch, T., ... Cardona, A. (2012). Fiji: An open-source platform for biological-image analysis. Nature Methods, 9(7), 676-682. https://doi. org/10.1038/nmeth.2019

Shen, K. Z., \& Johnson, S. W. (2006). Subthalamic stimulation evokes complex EPSCs in the rat substantia nigra pars reticulata in vitro. Journal of Physiology, 573(Pt 3), 697-709.

Wessel, J. R., \& Aron, A. R. (2017). On the globality of motor suppression: Unexpected events and their influence on behavior and cognition. Neuron, 93(2), 259-280. https://doi.org/10.1016/j.neuron.2016. 12.013

How to cite this article: Gouty-Colomer L-A, Michel FJ, Baude $A$, et al. Mouse subthalamic nucleus neurons with local axon collaterals. J Comp Neurol. 2018;526:275-284. https://doi.org/ 10.1002/cne.24334 